\title{
USABILIDADE PEDAGÓGICA - UMA DIMENSÃO PEDAGÓGICA NA PERSPECTIVA DA USABILIDADE DOS MOOCS
}

FLORIANÓPOLIS/SC ABRIL/2018

\author{
Vanessa Nascimento Mendes - UFSC - vvvmendes@gmail.com \\ Heluiza Ormelez de Almeida Nascimento - UFSC - heluiza@hotmail.com \\ Araci Hack Catapan - UFSC - aracihack@gmail.com
}

Tipo: Investigação Científica (IC)

Natureza: Planejamento de Pesquisa

Categoria: Métodos e Tecnologias

Setor Educacional: EDUCAÇÃO SUPERIOR, EDUCAÇÃO CORPORATIVA, EDUCAÇÃO CONTINUADA EM GERAL

\begin{abstract}
RESUMO
O presente artigo tem por objetivo apresentar alguns conceitos relacionados à usabilidade em sistemas voltados para o ensino da educação à distância (EaD) mediado pelo computador, mais especificamente no contexto dos MOOCs. Além de um panorama sobre a usabilidade, este artigo irá tratar especificamente de uma dimensão ou ampliação do conceito de usabilidade, a usabilidade pedagógica. A partir de uma análise qualitativa, apresenta-se neste artigo o conceito de usabilidade pedagógica, diferenciando-o de outros tipos de usabilidade, como a usabilidade de design e técnica, discute-se ainda sobre os testes de usabilidade pedagógica e as métricas envolvidas. Conclui tecendo considerações acerca do papel da usabilidade pedagógica, relacionando-a aos: objetivos educacionais, aspectos motivacionais, necessidades, experiências e expectativas dos alunos dos MOOCs.
\end{abstract}

Palavras-chave: Usabilidade. MOOC. Usabilidade Pedagógica

\section{AGRADECIMENTOS}

AS ACADÊMICAS VANESSA NASCIMENTO MENDES E HELUIZA ORMELEZ DE ALMEIDA NASCIMENTO AGRADECEM AO GRUPO DE PESQUISA - ATELIER TCD - TECNOLOGIA DE COMUNICAÇÃO DIGITAL PELO APORTE CIENTÍFICO. 


\section{INTRODUÇÃo}

O Nos últimos anos há um crescimento no número de usuários participando de MOOCs (Massive Open Online Courses). Segundo o New York Times, 2012 foi o ano do MOOC, um ano em que as pessoas começaram a se matricular de forma maciça e mundialmente em MOOCs, atingindo desta forma, um número de milhões de usuários dentro de apenas um período de cinco anos (PAPPANO, 2012).

A ideia central dos MOOCs consiste na oferta de cursos online que estão disponíveis gratuitamente para qualquer pessoa, a qualquer momento e em qualquer lugar. Sua utilização requer apenas acesso à Internet, uma conta de e-mail ou conta de uma rede social. Geralmente estes cursos são disponibilizados em plataformas que integram universidades que ofertam em massa cursos livres, normalmente sem custo, pagando apenas pela certificação.

Do ponto de vista socioeconômico, os MOOCs possibilitam aos seus usuários acesso à cursos voltados para atualização profissional, capacitação e ensino superior. Representam uma boa alternativa para pessoas que não possuem recursos econômicos para estudar em uma universidade ou faculdade.

Na literatura o termo "MOOC" está normalmente associado à promoção da educação aberta. Pireva, Imran e Dalipi (2015) apontam que o desenvolvimento dos MOOCs se baseia na filosofia de educação aberta, promovendo que o conhecimento deve ser compartilhado independentemente das restrições demográficas, econômicas, sociais e geográficas. Da mesma forma, Jiménez-González et al. (2016) descreve que o objetivo principal de um MOOC está relacionado ao acesso à educação. Para eles, o papel de um MOOC é fornecer acesso às pessoas que estão procurando concluir seus estudos e às pessoas que necessitam estender a sua educação, mas que de alguma forma não podem fazer isso presencialmente em salas de aula.

Segundo Siemens, Irvine e Code (2013) as pesquisas que estão surgindo têm ajudado professores e instituições a entender as vantagens dos MOOCs e suas limitações como ferramenta de ensino e aprendizagem. Para McAuley, Stewart, Siemens e Cormier (2010) os MOOCs apresentam para o cenário da educação desafios para os alunos, instrutores e instituições de ensino. De acordo com Yuan e Powell (2013) questões relacionadas à sustentabilidade (modelos de negócios), questões pedagógicas, qualidade e taxas de conclusão, representam os principais desafios para MOOCs.

Considerando o cenário apresentado, o presente artigo tem por objetivo resgatar alguns 
conceitos relacionados à usabilidade, aplicando-os em sistemas voltados para o ensino da educação à distância $(\mathrm{EaD})$ mediado pelo computador, mais especificamente no contexto dos MOOCs. Além de um panorama sobre a usabilidade, este artigo irá tratar especificamente de uma dimensão ou ampliação do conceito de usabilidade, a usabilidade pedagógica.

O conceito de usabilidade foi desenvolvido originalmente dentro da disciplina de IHC (Interação Humano-Computador) e aplicado à interação de um usuário com um computador. Conhecido como o pai da usabilidade, Nielsen (1993) afirmava que a usabilidade está relacionada à utilização de métodos que contribuam com a facilidade de uso durante o processo de criação de um produto. Para Nielsen (1993) a usabilidade não é uma propriedade única e unidimensional de uma interface de usuário; segundo o autor, a usabilidade possui múltiplos componentes e tradicionalmente está associada a esses cinco atributos:

Facilidade de aprendizagem: o sistema deve ser fácil de assimilar pelo utilizador, para que este possa começar a trabalhar rapidamente. Eficiência: o sistema deve ser eficiente para que o utilizador, depois de o saber usar, possa atingir uma boa produtividade. Facilidade de memorização: o sistema deve ser facilmente memorizado, para que depois de algum tempo sem o utilizar, o utilizador se recorde como usá-lo. Segurança: o sistema deve prever erros, evitar que os utilizadores os cometam e, se o cometerem, permitir fácil recuperação ao estado anterior. Satisfação: o sistema deve ser usado de uma forma agradável, para que os utilizadores fiquem satisfeitos com a sua utilização (Nielsen ,1993).

Normas como a ISO 9126 (1991) e a IS0 9241-11(2002) também apresentaram suas definições sobre a usabilidade. No caso da ISO 9126 (1991), é importante mencionar que o foco desta norma é a qualidade do produto de software, desta forma, propõe atributos de qualidade distribuídos em seis características: funcionalidade, confiabilidade, usabilidade, eficiência, manutenibilidade e portabilidade. Neste contexto, a usabilidade é entendida como um conjunto de atributos de software que se relaciona com o esforço requerido para seu uso por determinado conjunto de usuários (ISO 9126, 1991).

Para a ISO 9126 (1991) a usabilidade é definida pelos seguintes aspectos:

Inteligibilidade: atributos do software que evidenciam o esforço do usuário para reconhecer o conceito lógico e sua aplicabilidade. Apreensibilidade: identifica a facilidade de aprendizado do sistema para os seus potenciais usuários. 
Operacionalidade: atributos do software que evidenciam o esforço do usuário para sua operação e controle de sua operação. Atratividade: evidencia a satisfação subjetiva do usuário durante o uso. Conformidade: atributos do software que fazem com que o software esteja de acordo com as normas, convenções ou regulamentações previstas em leis e descrições similares, relacionadas à aplicação. Também significa que está em harmonia com padrões ou convenções relacionados à portabilidade (ISO 9126, 1991).

Para a IS0 9241-11(2002), norma apoiada nos requisitos ergonômicos, o conceito de usabilidade, é caracterizado como um conjunto de diretrizes que permitem ao usuário atingir seu objetivo e satisfação dentro de um contexto particular de necessidades. Quanto às definições apresentadas nesta norma tem-se:

Usabilidade: Medida na qual um produto pode ser usado por usuários específicos para alcançar objetivos específicos com eficácia, eficiência e satisfação, em um contexto específico de uso. Eficácia: Acurácia e completude com as quais usuários alcançam objetivos específicos. Eficiência: Recursos gastos em relação à acurácia e abrangência com as quais usuários atingem objetivos. Satisfação: Ausência do desconforto e presença de atitudes positivas durante o uso do produto. Usuário: Pessoa que interage com o produto. Objetivo: Resultado pretendido. Tarefa: Conjunto de ações necessárias para alcançar um objetivo (ISO 9241, 2002).

Corroborando com Nielsen (1994), Vetromille-Castro (2003) em seu estudo sobre a usabilidade e a elaboração de materiais para o ensino de inglês mediado por computador, define que a usabilidade é a medida do quão fácil é utilizar um produto para executar determinada tarefa. Este autor ainda afirma que no contexto de criação de software, o termo usabilidade representa uma abordagem que coloca o usuário, em vez de o sistema, no centro do processo. Nesta abordagem, chamada de design centrado no usuário, incorpora-se as preocupações e a defesa do usuário ao processo de design desde seu início e indica que as necessidades do usuário devem ser prioridade em qualquer decisão sobre esse design (VETROMILLE-CASTRO, 2003).

Além de definir a usabilidade no contexto do ensino mediado pelo computador, Vetromille-Castro (2003) traz para esta discussão a usabilidade vista sob duas perspectivas: usabilidade de design (UD) e usabilidade pedagógica (UP). Em suas constatações sobre a UD, o autor destaca o fato da uniformidade da interface facilitar a familiarização do aprendiz com o material. Para Vetromille-Castro (2003), o design de tela que o aluno encontra no início deve ser o mesmo que ele encontra ao final da atividade, propiciando uma aprendizagem do sistema mais rápida e o conseqüente aumento da confiança, segurança e orientação no novo meio. 
Quanto à a usabilidade pedagógica, Vetromille-Castro (2003) afirma que ela está focalizada no fornecimento de feedback e estratégias de leitura, e que uma baixa usabilidade pedagógica pode prejudicar os processos de aprendizagem. $\mathrm{O}$ autor aponta que a UP tem relação mais restrita com a atividade proposta e que o material didático deve ser desenvolvido para o público pretendido. Esse material deve ser relevante e motivador, possibilitando que os alunos possam se envolver amplamente com as atividades e obter o resultado esperado: a aprendizagem.

Em seu outro estudo sobre a usabilidade pedagógica e de design de materiais para o ensino a distância de Inglês para leitura, Vetromille-Castro (2002) também caracteriza a UP relacionando-a ao papel do feedback na aprendizagem. Para o autor, o uso da língua materna na atividade, o tipo de feedback fornecido ao aluno, o momento em que o feedback deve ser dado, o tipo de atividade proposta, entre outros aspectos pedagógicos, constituiria um novo viés da usabilidade, a usabilidade pedagógica.

\section{USABILIDADE PEDAGÓGICA}

Com o objetivo de compreender o conceito e o papel da usabilidade pedagógica, serão aqui apresentados alguns autores que caracterizam a usabilidade no contexto do ensino mediado por sistemas e plataformas online. Kukulska-Hulme \& Shield (2004) ao explorarem a usabilidade dos sites de e-learning, com referência especial a aprendizagem de línguas estrangeiras, mostram que há quatro camadas na compreensão do conceito de usabilidade pedagógica: a camada contextual, a acadêmica, a geral e a técnica. A usabilidade contextual se relaciona a cursos e a disciplinas específicas; a usabilidade acadêmica trata de estratégias pedagógicas e questões educacionais; a usabilidade geral é comum à maioria dos websites e pressupõe fatores como clareza na navegação e acessibilidade; e por fim, a usabilidade técnica trata de questões objetivas, como links quebrados e capacidade de resposta do computador servidor.

Para Agner (2009) a usabilidade pedagógica pode envolver o estudo de questões mais avançadas de teorias educacionais e tem uma experiência bem-sucedida em pesquisa de avaliação de usabilidade, com o objetivo de cooperar na consolidação do conceito.

Sales Junior (2016) traz para a discussão sobre a usabilidade pedagógica, o texto da ISO 9241-11. Segundo o autor, entende-se que a usabilidade pedagógica pode ser avaliada no contexto do ambiente educacional, ocasião em que o conteúdo é 
consumado por estudantes específicos, que desejam alcançar objetivos educacionais específicos de forma efetiva e eficiente, e ficando satisfação em sua aprendizagem. Portanto, a usabilidade pedagógica é estudada quando retorna a um ambiente de ensino. Caso a interação entre alunos e sistemas que utilizem tecnologias ocorra apenas para uma transmissão unidirecional de instruções, os artefatos existentes disponíveis para mudar fundamentalmente as concepções e os métodos de ensinoaprendizagem serão subutilizados (SALES JUNIOR, 2016).

Assim como Sales Junior (2016), outros autores também associam a usabilidade pedagógica ao alcance de objetivos educacionais. Ao avaliar o impacto da usabilidade técnica e pedagógica no desempenho de aprendizes em e-learning, Reitz (2009) analisa que os aspectos de usabilidade apresentam uma dimensão adicional para os ambientes educacionais, visto que garantir que um sistema de e-learning seja usável já não é o suficiente.

Este sistema deve também ser efetivo no sentido de se adequar aos objetivos pedagógicos. Ao avaliar a usabilidade de uma interface web móvel de um LMS, Ivanc, Vasiu e Onita (2012) caracterizam a usabilidade pedagógica como a análise da forma como uma aplicação educacional (ferramentas, conteúdo, tarefas e interface) auxilia os alunos no seu processo de aprendizagem em vários contextos de aprendizagem de acordo com os objetivos de aprendizagem. Para Ivanc, Vasiu e Onita (2012) a usabilidade pedagógica deve ser especialmente preocupada com os aspectos educacionais, como o processo de aprendizagem, os fins de aprendizagem, as necessidades do usuário, a experiência de aprendizagem, o conteúdo de aprendizagem e os resultados de aprendizagem.

Além de contribuírem para com o conceito da usabilidade pedagógica, Kukulska-Hulme \& Shield (2004) ressaltam sua importância; segundo os autores, esse atributo afeta o design e o desenvolvimento do site educacional, particularmente no contexto da educação aberta e a distância. Relacionando a usabilidade pedagógica com a usabilidade geral, Kukulska-Hulme \& Shield (2004) afirmam que independentemente do grau de eficácia pedagógica do conteúdo ou sistema, será pouco útil se o aluno não conseguir localizá-lo em um sistema mal organizado. Sendo assim, a usabilidade pedagógica é baseada em princípios de usabilidade geral.

Silius e Tervakari (2003) usam o termo para questionar se as ferramentas, o conteúdo, a interface e as tarefas de ambientes baseados na web atendem às necessidades de aprendizagem de diferentes alunos, em muitos contextos, de acordo com os objetivos educacionais especificados. Para estes autores, existem diversas formas de 
implementar e avaliar os requisitos de usabilidade pedagógica, e o teste de usabilidade é um deles.

Quanto aos testes de usabilidade, Vetromille-Castro (2003) afirma que eles são feitos em softwares que se concentram apenas na interface, na sua aparência e como o usuário se orienta para navegar. Esse tipo de teste é fundamental, mas percebe-se ser essencial, também, avaliar características que envolvam mais que a apresentação do material, mas também como foi conduzida a sua preparação pedagógica. Assim, acredita-se que uma característica essencial para que o curso tenha uma alta UP está relacionada ao feedback e como o aluno é assistido tanto pelo sistema como pelo professor e também o suporte técnico (VETROMILLE-CASTRO, 2003).

Em seu estudo sobre usabilidade e a importância de testar interfaces para o ensino a distância mediado pelo computador, Martins e Barbosa (2006) afirmam que a adequabilidade do sistema ao aluno a que se destina pode ser verificada através da mensuração do seu nível de usabilidade, ou seja, através de testes de usabilidade.

Para Martins e Barbosa (2006) existem dois tipos de avaliação que precisam ser realizadas para conferir se o sistema promoverá o sucesso desejado: os testes de Usabilidade de Design (UD), que avaliam as escolhas e a qualidade tecnológica dos ambientes educacionais, e os testes de Usabilidade Pedagógica (UP) que examinam se as opções didáticas e metodológicas ofertadas aos alunos/usuários por meio de atividades e tarefas promovem de fato a aprendizagem.

Em sua pesquisa sobre critérios de usabilidade para a avaliação de material na aprendizagem digital, Nokelainen (2006) definiu a usabilidade pedagógica como sendo um conjunto de critérios pedagógicos que podem ser aplicados a qualquer material de aprendizagem digital, como controle de alunos, aprendizado colaborativo, valor agregado, motivação, flexibilidade, feedback, etc. Ainda segundo este autor, a usabilidade pedagógica de um sistema e / ou material de aprendizagem depende dos objetivos estabelecidos para uma situação de aprendizagem pelo aluno e professor. Ao avaliarem a usabilidade do LMS Moodle; Ivanc, Vasiu e Onita (2012) também definiram algumas métricas relevantes para a usabilidade pedagógica, são elas: instrução, aprender, conteúdo, relevante, estrutura de conteúdo de aprendizagem, tarefas, variáveis do aluno, aprendizado colaborativo, facilidade de uso, controle do aluno e motivação.

Além da definição de critérios de usabilidade, Nokelainen (2006) contribui para esta discussão por meio de sua adaptação do sistema de aceitabilidade proposto por Nielsen 
(1993). Nesta adaptação o autor apresentou dois atributos de usabilidade não contemplados por Nilesen (1993): usabilidade técnica e usabilidade pedagógica. Neste contexto, a usabilidade pedagógica é um subconjunto do critério utilidade, enquanto que usabilidade técnica é um subconjunto de usabilidade.

Hadjerrouit (2010) ao estudar sobre o desenho e a avaliação de recursos de aprendizagem digital, o qual chamou de WBLR (recursos de aprendizagem baseados na Web) aponta que o design deste tipo de recurso é ainda de domínio de especialistas técnicos e de software, em vez de professores, educadores e aprendizes. Hadjerrouit (2010) enfatiza que a maioria dos WBLRs basicamente enfatizam a usabilidade técnica, para o autor a usabilidade técnica serve para minimizar a carga cognitiva e ajuda a liberar mais recursos para o próprio processo de aprendizagem, contribui para que o aluno se concentre mais facilmente nos materiais de aprendizagem sem ter que realizar um esforço para descobrir como acessá-los.

Embora Hadjerrouit (2010) reconheça a utilidade e importância da usabilidade técnica, alerta que ela não é suficiente para garantir o aprendizado e sugere que os desenvolvedores precisam projetar WBLRs com uma nova dimensão de usabilidade, a usabilidade pedagógica. Segundo o autor este tipo de usabilidade está associado a aspectos fundamentais para a aprendizagem, e adverte que do ponto de vista da usabilidade pedagógica, os WBLRs atuais não possuem uma série de características que os tornariam mais flexíveis, interativos, motivadores e colaborativos.

Para Hadjerrouit (2010), a usabilidade técnica envolve técnicas para garantir uma interação sem problemas com o software, enquanto que a usabilidade pedagógica visa apoiar o processo de aprendizagem dos alunos. Assim como Hadjerrouit (2010), Reitz (2009) diferencia os conceitos de usabilidade pedagógica e técnica. Para a autora, a usabilidade técnica diz respeito aos critérios e recomendações de usabilidade que apresentam como objetivo facilitar o uso e a eficiência das funções, dos objetos de interação e das características das interfaces melhorando a interação dos alunos para com as interfaces do material de aprendizagem. Já a usabilidade pedagógica define os critérios e recomendações de usabilidade que visam satisfazer as necessidades dos alunos para a realização das tarefas ou atividades propostas por meio dos elementos da interface do material de aprendizagem (REITZ,2009).

\section{CONSIDERAÇÕES FINAIS}


Devido à popularização e a crescente oferta da $\mathrm{EaD}$, e o uso cada vez mais frequente de softwares e plataformas web voltadas para a educação, um dos grandes desafios da atualidade é o de superar os problemas de usabilidade que os dispositivos móveis e interfaces da web vem revelando. Por meio deste artigo, tem-se que a usabilidade é um dos principais fatores de sucesso de um sistema ou plataforma educacional, desta forma, é fundamental que se alcance um bom nível de usabilidade; que conforme aqui apresentado, a usabilidade pode ser analisada sob diversas perspectivas: design centrada no usuário, técnica e pedagógica.

A usabilidade pedagógica difere das demais perspectiva, pois foca em elementos relacionados à motivação, necessidades, experiências e expectativas dos alunos, também aqui considerado de usuários. Nesta perspectiva, o fornecimento do feedback tem seu papel relevante, pois proporciona ao aluno o aumento da confiança e segurança, oportunizando uma aprendizagem rápida.

Muito além de uma facilidade de uso e de navegação, a usabilidade pedagógica tem como objetivo satisfazer as necessidades dos alunos para a realização de tarefas e atividades propostas. Assim como o conteúdo apresentado, estas atividades devem ser relevantes ao aluno e alinhadas aos objetivos educacionais.

\section{REFERÊNCIAS}

AGNER, Luiz Carlos Caldas. Inovação e qualidade do design na educação online: uma contribuição à usabilidade pedagógica. In: 15. Congresso Internacional ABED de Educação a Distância, 2009, Fortaleza, CE. Anais do 15 CIAED. São Paulo, SP: ABED, 2009.

G. SIEMENS, V. IRVINE, and J.CODE, "Guest Editors' Preface to the Special Issue on MOOCs: An Academic Perspective on an Emerging Technological and Social Trend," MERLOT Journal of Online Learning and Teaching, vol. 9, no. 2, pp. iii-vi, Jun. 2013; http://jolt.merlot.org/vol9no2/siemens_editorial_0613.htm

HADJERROUIT, S. (2010). A conceptual framework for using and evaluating Webbased learning resources in school education. Journal of Information Technology Education, 9, 53-79. Available at http://www.jite.org/documents/Vol9/JITEv9p053-079Hadjerrouit743.pdf 
ISO 9126: Software product evaluation - Quality characteristics and guidelines for their use, 1991.

ISO 9241. Ergonomic Requirements for office work with visual display terminals. Part 10: Dialogue Principles; Draft International Standard ISO, 1996.

IVANC, D., VASIU, R., \& ONITA, M. (2012). Usability Evaluation of a LMS Mobile Web Interface. Information and Software Technologies, 319(2), 348-361.

KUKULSKA-HULME, A.; SHIELD, L. The keys to usability in e-learning Websites. The Open University, 2004. Disponível em: http://www.networkedlearningconference.org. uk/past/nlc2004/proceedings/individual_papers/ kukulska_shield.htm. Acesso em: jul. 2008.

MARTINS, Maria de Lourdes Oliveira; BARBOSA, Ana Cristina Chagas. Usabilidade: a importância de testar interfaces para o ensino a distância mediado pelo computador. In: SEMINÁRIO NACIONAL DE EDUCAÇÃO A DISTÂNCIA, 2006, Brasília. Anais... p. 1-13, 2006. Disponível em: http://www.abed.org.br/seminario2006/pdf/tc031.pdf. Acesso em: 1ํ jun. 2017.

McAuley, A.; Stewart, B.; Siemens, G. \& Cormier, D. (2010). The MOOC Model for Digital Practice: Massive Open Online Courses. Digital ways of knowing and learning. University of Prince Edward Island. Recuperado el 6 de Julio de 2013. https://oerknowledgecloud.org/sites/oerknowledgecloud.org/files/MOOC_Final_0.pdf

NIELSEN, Jakob. Heuristic evaluation. In Nielsen, J., and Mack, R.L. (Eds.), Usability Inspection Methods, John Wiley \& Sons, New York, NY, 1994.

. Usability Engineering. Boston, USA: Academic Press, Inc., 1993.

NOKELAINEN, Petri. An empirical assessment of pedagogical usability criteria for digital learning material with elementary school students. Educational Technology \& Society, v. 9 (2), p. 178-197, 2006.

PAPPANO, L. (2012, Nov. 2). Year of the MOOC, New York Times. Retrieved from Retrievedfromhttp://www.nytimes.com/2012/11/04/education/edlife/massive-open-onlinecourses-are-multiplying-at-a-rapid-pace.html?pagewanted=all\&_r=0. 